\title{
Trans-anal reinforcing sutures after double stapling for lower rectal cancer: Report of two cases
}

\author{
MASAYA MUKAI ${ }^{1}$, MAKI FUKASAWA ${ }^{1}$, KYOKO KISHIMA ${ }^{1}$, SHIN-ICHI IIZUKA ${ }^{1}$, HIROSHI FUKUMITSU ${ }^{1}$, \\ NAOKI YAZAWA ${ }^{1}$, TAKAYUKI TAJIMA ${ }^{1}$, MASATO NAKAMURA ${ }^{2}$ and HIROYASU MAKUUCHI ${ }^{3}$ \\ ${ }^{1}$ Department of Surgery, Tokai University Hachioji Hospital, Ishikawa-cho 1838, Hachioji, Tokyo 192-0032; \\ ${ }^{2}$ Departments of Pathology, ${ }^{3}$ Surgery, Tokai University School of Medicine, Bohseidai, Isehara, Kanagawa 259-1193, Japan
}

Received August 19, 2008; Accepted November 14, 2008

DOI: 10.3892/or_00000226

\begin{abstract}
To safely avoid the construction of a covering stoma in patients with advanced lower rectal cancer undergoing laparoscopy assisted colorectal surgery (LACS), we added circumferential manual reinforcing sutures via the transanal approach at the site of mechanical anastomosis. In June 2008, LACS was performed for a tumor of $6 \mathrm{~cm}$ in longer diameter in the $\mathrm{Rb}$ region of the lower rectum $\sim 5 \mathrm{~cm}$ from the anal verge. After intraperitoneal coloproctal anastomosis was performed in the pelvis by the double stapling technique (DST), reinforcement was provided by manual trans-anal suturing (trans-anal reinforcing sutures: TARS). A covering stoma was constructed because this was a high-risk case. Complications such as mild wound infection and stoma trouble occurred, and the patient was discharged after conservative therapy. In June 2008, LACS was performed for a tumor of $5 \mathrm{~cm}$ in longer diameter in the Ra region of the lower rectum $\sim 7 \mathrm{~cm}$ from the anal verge. After intraperitoneal colorectal anastomosis was performed in the pelvis by DST, TARS were added to avoid a covering stoma. Minor leakage occurred postoperatively, but this was controlled conservatively and the patient was discharged. In patients having surgical treatment of advanced lower rectal cancer, good results were obtained by adding circumferential reinforcing sutures via the trans-anal approach at the site of ultra-low anastomosis after DST.
\end{abstract}

Correspondence to: Dr Masaya Mukai, Department of Surgery, Tokai University Hachioji Hospital, Ishikawa-cho 1838, Hachioji, Tokyo 192-0032, Japan

E-mail: mukai.masaya@hachioji-hosp.tokai.ac.jp

Abbreviations: TARS, trans-anal reinforcing sutures; DST, double stapling technique; LAR, low anterior resection; LACS, laparoscopy assisted colorectal surgery

Key words: low rectal cancer, double stapling technique, low anterior resection, trans-anal reinforcing sutures, laparoscopy assisted colorectal surgery, covering stoma

\section{Introduction}

In recent years, less invasive laparoscopy-assisted colorectal surgery (LACS) has become very common, and the range of indications for such surgery has broadened markedly to include additional resection for stage I/early colorectal cancer, curative resection of advanced stage II/III cancer, and palliative procedures for stage IV cancer (1-5). However, in patients with lower rectal cancer located close to the anus, a covering stoma of the temporary loop ileostomy or loop colostomy is often created to prevent fatal complications, such as disruption of sutures at the ultra-low anastomosis, even in patients having routine standard laparotomy (6-8). When LACS is performed, a covering stoma is still often constructed, e.g., 1) when the double stapling technique (DST) is applied after multiple mechanical transection of the lower rectum, 2) if there is high tension at the site of anastomosis after pull-through of the colon, or 3) if there is poor blood supply to the colon on the oral side of the anastomosis.

Construction of a loop stoma imposes a considerable mental and physical burden on patients irrespective of whether they have undergone standard laparotomy or LACS. Since minimally invasive surgery is the fundamental reason for selecting LACS, loop stoma construction should be avoided whenever possible, even in patients with lower rectal cancer. However, the actual frequency of covering stoma construction is relatively high, presumably because pelvic peritonitis due to suture disruption is potentially fatal and because extremely complicated manipulations are required to achieve anastomosis when treating advanced lower rectal cancer in elderly patients with concurrent diseases or male patients with a narrow pelvis and high body mass index (BMI) (6-8).

In order to safely avoid the construction of a covering stoma in patients with advanced lower rectal cancer undergoing LACS, we added circumferential manual reinforcing sutures via the transanal approach (transanal reinforcing sutures: TARS) at the site of mechanical anastomosis after performing intraperitoneal ultra-low anastomosis by the usual DST method in the pelvic floor and obtained favorable results.

\section{Case reports}

Case 1. A 47-year-old unmarried man was referred to our hospital due to bloody stools. He had a history of head surgery 


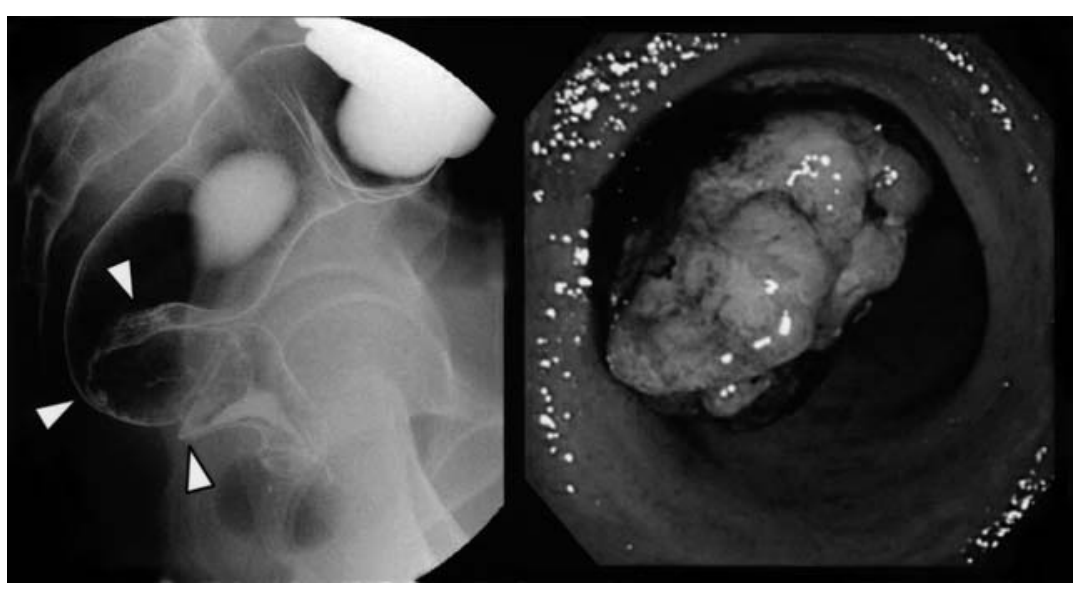

Figure 1. Barium enema reveals a type 2 cancer of $6 \mathrm{~cm}$ in longer diameter in the $\mathrm{Rb}$ region of the lower rectum $\sim 5 \mathrm{~cm}$ from the anal verge (left, arrowheads). Tumor biopsy at colonoscopy discloses moderately differentiated adenocarcinoma (right).

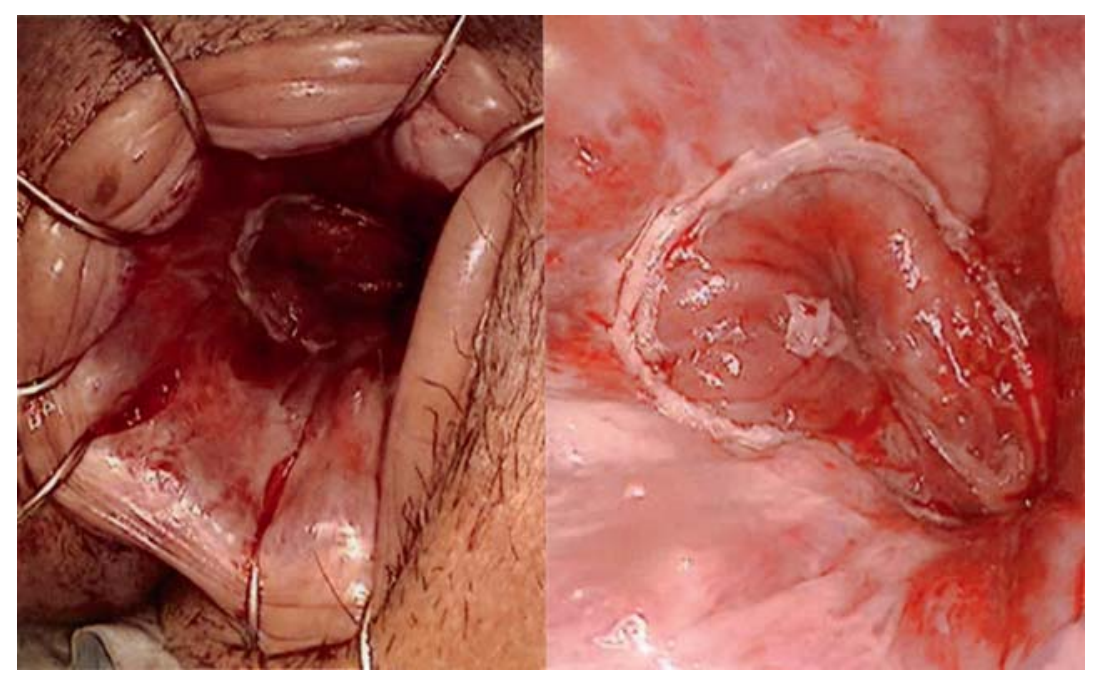

Figure 2. The transanal approach is facilitated with an anal retractor (left). Coloproctal mechanical anatomosis can be observed circumferentially just above the dentate line (right).

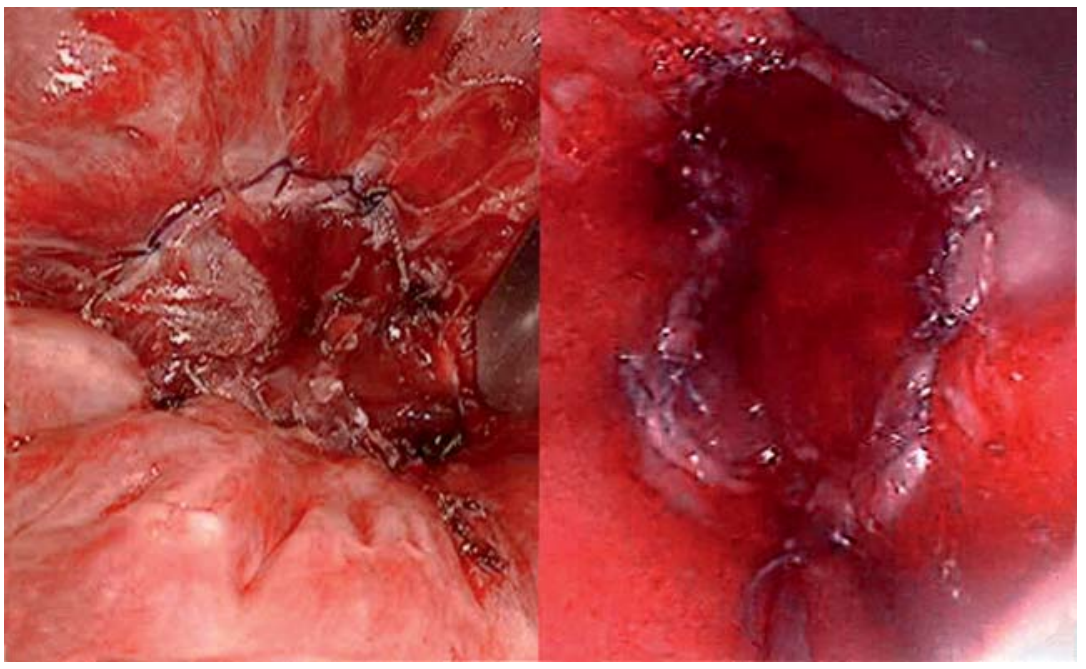

Figure 3. Case 1: Trans-anal reinforcing sutures (TARS). Continuous suturing with absorbable 3-0 PDS-II attached to a needle is being mainly done on the upper site of the mechanical anastomosis (left). Case 2: Trans-anal reinforcing sutures (TARS). Interrupted sutures are mainly being placed in the upper site to the mechanical anastomosis with absorbable 3-0 Vicryl attached to a needle (right). 


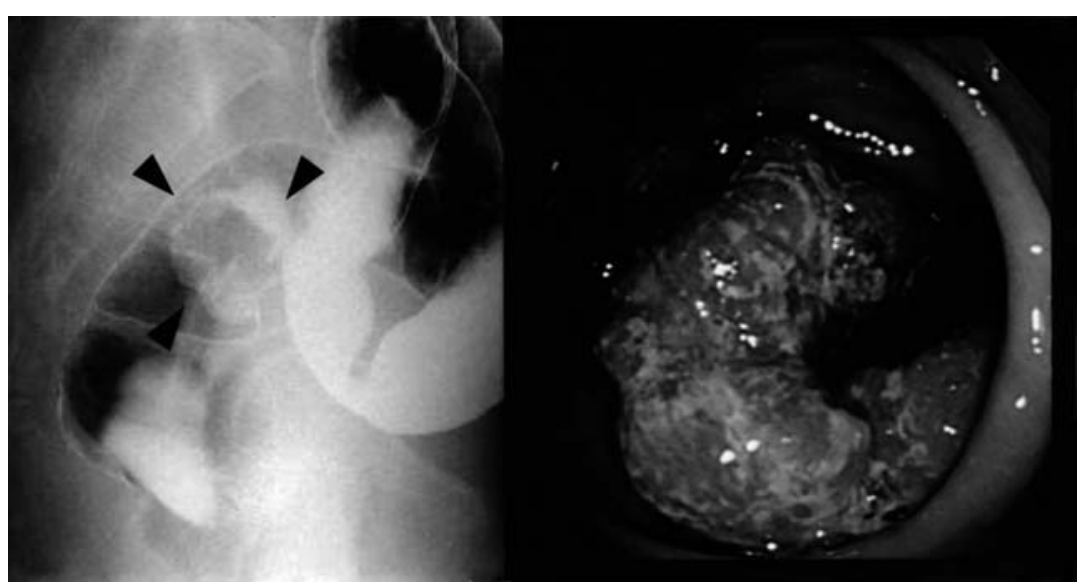

Figure 4. Barium enema reveals a type 2 cancer of $5 \mathrm{~cm}$ in longer diameter in the Ra region of the lower rectum $\sim 7 \mathrm{~cm}$ from the anal verge (left, arrowheads). Tumor biopsy at colonoscopy discloses well differentiated adenocarcinoma (right).

because of subarachnoid hemorrhage and a pacemaker had been inserted to control arrhythmia and heart failure. Barium enema (Fig. 1; left, arrow heads) and colonoscopy revealed a tumor of $6 \mathrm{~cm}$ in longer diameter in the $\mathrm{Rb}$ region of the lower rectum $\sim 5 \mathrm{~cm}$ from the anal verge, and biopsy gave a diagnosis of moderately differentiated adenocarcinoma (Fig. 1; right). It was difficult to persuade the patient to undergo temporary loop stoma construction, and he refused to undergo Miles' operation with a higher risk of sexual dysfunction than low anterior resection (LAR). However, informed consent to these procedures was eventually obtained from the patient, and he was hospitalized to undergo LACS-LAR or LACSMiles' operation. Based on the results of various preoperative examinations, the Department of Anesthesiology stated that this patient was a high-risk case because his past history included two major diseases. In June 2008, he underwent LACS-LAR (hybrid 3-port HALS D2 operation), while Miles' operation associated with permanent endocolostomy was avoided in consideration of his strong preoperative request (5). An adequate distal safety margin $(\geq 2 \mathrm{~cm})$ was obtained on the anal side of the tumor. After irrigation, the rectum was completely transected twice with an Endopath stapler, Ethlon $60 \mathrm{~mm}$-Gold cartridge (Ethicon Endo-Surgery, Puerto Rico, USA). Subsequently, using a Proxymate ILS Endopath, Endo circular stapler (ECS33; Ethicon Endo-Surgery), intraperitoneal anastomosis was performed in the pelvis by coloproctal mechanical suturing according to the standard double stapling technique (DST) (Fig. 2; right). Then an anal retractor (The Lone Star Retractor System, Yufu Itonaga Co., Ltd., Tokyo, Japan) was inserted (Fig. 2; left), and circumferential continuous transanal reinforcing sutures (TARS) were added with absorbable 3-0 PDS-II attached to a needle (Ethicon Inc., NJ, USA) at the site of ultra-low anastomosis by the transanal approach (Fig. 3; left). Since this was an extremely high-risk case, a temporary covering right transverse loop colostomy was also created. The tumor was an advanced lower rectal cancer located in the $\mathrm{Rb}$ region, which was classified as P0H0N1A2 (intraoperative frozen section; distal anal margin, cancer negative) and pathological stage III. There were mild wound infection and no sexual dysfunction with normal erection and ejaculation. Self-management of the stoma also became possible, and the patient was discharged from hospital at 27 days postoperatively.

Case 2. A 63-year-old woman was referred to our hospital with melena. Barium enema (Fig. 4; left, arrow heads) and colonoscopy revealed a tumor of $5 \mathrm{~cm}$ in longer diameter in the Ra region of the lower rectum $\sim 7 \mathrm{~cm}$ from the anal verge, and biopsy gave a diagnosis of well differentiated adenocarcinoma (Fig. 4; right). At first, the patient strongly refused to undergo construction of a temporary loop stoma, even in case of emergency, but informed consent was finally obtained and she was hospitalized for LACS. Based on the results of various preoperative examinations, severe diabetes was noted as a risk factor. LACS-LAR (hybrid 3-port HALS D2 operation) was performed in June 2008 (5). An adequate distal safety margin $(\geq 2 \mathrm{~cm})$ was achieved on the anal side of the tumor. After irrigation, the rectum was completely transected with a 51-mm curved cutter, Green-cartridge (Ethicon Endo-Surgery). To avoid the need for a covering loop stoma, as was strongly desired by the patient, an anal retractor (The Lone Star Retractor System, Yufu Itonaga Co.) was inserted and circumferential interrupted transanal reinforcing sutures (TARS) were added with absorbable 3-0 Vicryl attached to a needle (Ethicon Inc.) (Fig. 3; right). The tumor was an advanced lower rectal cancer located in the $\mathrm{Ra}$ region, which was classified as P0H0NOA2 (intraoperative frozen section; distal anal margin, cancer negative) and pathological stage II. Although minor leakage occurred postoperatively, it was controlled by conservative therapy without stoma construction. She was discharged from hospital at 19 days postoperatively with well controlled diabetes and good anal function with bowel passage.

\section{Discussion}

In recent years, ultra-low anastomosis that preserves anal function has been performed actively for the treatment of lower rectal cancer (1-5). The rectal prolapse method is also available for this purpose, in which the reversed rectum is pulled out of the pelvic cavity through the anus and transected extracorporeally, after which reconstruction is done by DST. 
However, this procedure is not indicated for patients with advanced lower rectal cancer and large tumors, because it is often difficult to pull the reversed rectum through the anus in patients with larger tumors or abundant mesorectal adipose tissue $(9,10)$. When jejunal J-pouch reconstruction is done after total resection of the large bowel, the pouch is usually pulled into the anal canal and attached by a single layer of fullthickness transanal sutures. However, this method goes against the fundamental concept that stoma construction should be avoided if possible, because a covering stoma is essential after full-thickness single-layer suturing. We routinely inject air into the anastomotic site/rectum when DST reconstruction is done after LAR and perform an air leak test with water in the pelvis. We observed tiny air bubbles rising from the anastomotic site where staples were slightly loose because of inadequate stapler closure or through pin-holes formed at the site of staple insertion. In such cases, full-thickness interrupted reinforcing sutures covering the stapled site are added to avoid severe complications. We have also performed loop sigmoid colostomy and transanal fistula closure to manage urethrorectal fistulae arising after high-intensity focused ultrasound (HIFU) therapy for prostate cancer $(11,12)$. If this trans-anal approach is learned properly, it becomes possible to perform two-layer suturing of the prostatic urethra and rectal mucosa to cover a prostatic urethral fistula in the rectum $\sim 6-7 \mathrm{~cm}$ from the anal verge. Based on these experiences, we developed TARS in which full-thickness/circumferential reinforcing sutures are added manually after mechanical suturing by DST. Leakage from ultra-low anastomoses may be ascribable to small holes that are formed in the vicinity of the perineal anastomosis by the effect of tension when the patient adopts the sitting position early after surgery or anastomotic rupture due to a rapid increase of intrarectal pressure caused by excessive retention of gas. Reinforcement of the anastomosis is considered to be the most effective countermeasure for these physical/mechanical factors.

When lower rectal anastomosis is performed below the peritoneal reflection, pelvic floor peritonitis due to suture disruption is a potential serious complication that every gastroenterological surgeon encounters at least once (13). It is also well-known that the septic state tends to improve soon after loop stoma construction, although when and where the stoma should ideally be constructed remain questions to be answered. It has sometimes been advocated that a preventive diverting loop stoma should be constructed after lower rectal anastomosis (14-16). In Western countries, radiation therapy is often performed both preoperatively and postoperatively in patients with advanced rectal cancer (17-19). On the other hand, it has long been considered in Japan that bilateral lymph node dissection should be done for lateral lymph node metastasis (20-22). There have been many studies that the outcome was better when lateral lymph node dissection was performed in patients with lateral lymph node metastasis. However, there is still controversy about the efficacy of prophylactic lateral lymph node dissection. Therefore, although the indications for LACS in Japan have increased markedly to include stage I-IIIa (N1 lymph node metastasis involving $\leq 3$ nodes) and palliative stage IV surgery depending on the criteria of each institution, there is still controversy about the need for prophylactic bilateral lymph node dissection in patients without obvious lateral lymph node metastasis preoperatively (20-22). We perform hybrid 3-port HALS as the standard operation, and there is no difference in layer separation from standard laparotomy if the left hand can reach the posterior surface of the tumor (5). Moreover, manipulations at the pelvic floor, which are usually performed under nearly blind conditions because of difficulty in observation, can be done safely under magnification and monitoring. Therefore, it seems possible to perform LACS in most patients.

Of course, the absence of stoma is more comfortable for patients. However, even when standard laparotomy is done, there are some patients who need covering loop stoma construction for various reasons. Therefore, the argument that covering stoma construction should be avoided absolutely if LACS is performed as minimally invasive surgery cannot be accepted. Furthermore, in patients with advanced lower rectal cancer, metastasis/recurrence may occur in the pelvic cavity relatively soon after covering stoma construction, e.g., at the site of anastomosis or on the anterior surface of the sacrum, while there are patients in whom chemoradiotherapy can be performed more efficiently because of loop stoma construction. Apart from patients who will receive no appreciable benefit from covering stoma closure or who want to avoid surgical stress, poor-prognosis patients with distant metastasis/recurrence in the liver or lungs and elderly patients with con-current diseases, generally undergo stoma closure at 3-6 months postoperatively (6). Considering its fundamental concept, it is evident that absence of a stoma is the best outcome for LACS, even more than for standard laparotomy, and therefore we should try to avoid stoma construction where possible. In the future, we need to obtain long-term follow-up data in a larger number of patients having TARS after DST to assess postoperative complications such as anal function, and the local recurrence rate at the anastomotic site or on the anterior sacrum.

\section{Acknowledgements}

This study was supported by grants from the Occult Neoplastic Cells Research and Study Group (\#2008-5007; Tokai University Hachioji Hospital, Tokyo, Japan) and the Research and Study Program of Tokai University Educational System General Research Organization (\#2007-04; Tokai University School of Medicine, Kanagawa, Japan).

\section{References}

1. Mukai M, Tokunaga N, Ishida H, Makukuchi H, Tajima T and Mitomi T: Clinical experiences with laparoscopic colectomy. Dig Endosc 9: 11-15, 1997.

2. Mukai M, Okamoto Y, Oida Y, Mukoyama S, Ito I, Nakasaki H, Kawai K, Sato S and Makuuchi H: Endoscopic mucosal resection for superficially spreading colonic neoplasms larger than $5 \mathrm{~cm}$ in the right colon after dilute sodium hyaluronate injection: Report of two cases. Endoscopy 35: 973-975, 2003.

3. Knack \& Pit Falls: Surgery of the colon, rectum and anus (2nd edition), Bunkoudou, Co., Ltd., Tokyo, 2004.

4. Guide line of large Bowel Cancer in Japan: Japanese Society for Cancer of the Colon and Rectum, Tokyo, 2005.

5. Mukai M, Tanaka A, Tajima T, Yamagiwa T, Okada K, Fukasawa M, Sato K, Oida Y, Nakamura M and Makuuchi H: Two-port hand-assisted laparoscopic surgery (HALS) in 2-stage treatment for complete obstruction by left colon cancer: A case report. Oncol Rep 19: 875-879, 2008. 
6. Mukai M, Himeno S, Mukoyama S, Tajima T, Saito Y, Ito I, Nakasaki H, Sato $\mathrm{S}$ and Makuuchi H: Is the temporally loop colostomy in the right transverse colon appropriate for complete obstruction by colorectal cancer? Oncol Rep 10: 693$698,2003$.

7. Gooszen AW, Geelkerken RH, Hermans J, Lagaay MB and Gooszen HG: Temporary decompression after colorectal surgery: randomized comparison of loop ileostomy and loop colostomy. Br J Surg 85: 76-79, 1998.

8. Rullier E, Le Toux N, Laurent C, Garrelon J-L, Parneix M and Saric J: Loop ileostomy versus loop colostomy for defunctioning low anastomoses during rectal cancer surgery. World J Surg 25: 274-278, 2001.

9. Fukunaga M, Kidokoro A, Iba T, Sugiyama K, Fukunaga T, Nagakari K, Suda M and Yoshikawa S: Laparoscopy assisted low anterior resection with a prolapsing technique for low rectal cancer. Surg Today 35: 598-602, 2005.

10. Idani $\mathrm{H}$, Ishii $\mathrm{T}$, Maruyama $\mathrm{M}$, Ishikawa $\mathrm{T}$, Iwamoto $\mathrm{T}$ and Watanabe S: Laparoscopic low anterior resection using a prolapsing technique for low rectal cancer. Surgery 67: 1359-1362, 2005 (In Japanese).

11. Uchida T, Ohkusa H, Nagata Y, Hyodo T, Satoh T and Irie A: Treatment of localized prostate cancer using high-intensity focused ultrasound. BJU Int 97: 56-61, 2006.

12. Uchida T, Ohkusa H, Yamashita H, Shoji S, Nagata Y, Hyodo T and Satoh T: Five years experience of transrectal high-intensity focused ultrasound using the sonablate device in the treatment of localized prostate cancer. Int J Urol 13: 228-233, 2006.

13. Karanjia ND, Corder AP, Holdsworth PJ and Heald RJ: Risk of peritonitis and fatal septicaemia and the need to defunction the low anastomosis. Br J Sur 78: 196-198, 1991.

14. Pakkastie TE, Ovaska JT, Pekkara ES, Luukkonen PE and Jarvinen HJ: A randomized study of colostomies in low rectal anastomoses. Eur J Sur 163: 929-933, 1997.
15. Dehni N, Schlegel RD, Cunningham C, Guiguet M, Tiret E and Pare R: Influence of a defunctioning stoma on leakage rates after low colorectal anastomosis and colonic J pouch-anal anastomosis. Br J Sur 85: 1114-1117, 1998

16. Tschmelitsch J, Wykypiel H, Prommegger R and Bodner E: Colostomy vs. tube cecostomy for protection of low anastomosis in rectal cancer. Arch Surg 134: 1385-1388, 1999.

17. NIH consensus conference: Adjuvant therapy for patients with colon and rectal cancer. JAMA 264: 1444-1450, 1990

18. Krook JE, Moertel CG, Gunderson LL, Wieand HS, Collins RT, Beart RW, Kubista TP, Poon MA, Meyers WC, Mailliard JA, Twito DI, Morton RF, Veeder MH, Witzig TE, Cha S and Vidyarthi SC: Effective surgical adjuvant therapy for high-risk rectal carcinoma. N Engl J Med 324: 709-715, 1991.

19. Wolmark N, Wieand HS, Hyams DM, Colangelo L, Dimitrov NV, Romond EH, Wexler M, Prager D, Cruz AB Jr, Gordon PH, Petrelli NJ, Deutsch M, Mamounas E, Wickerham DL, Fisher ER, Rockette H and Fisher B: Randomized trial of post operative adjuvant chemotherapy with or without radiotherapy for carcinoma of the rectum: National Surgical Adjuvant Breast and Bowel Project Protocol R-02. J Natl Cancer Inst 92: 388-396, 2000.

20. Moreira LF, Hizuta A, Iwagaki H, Tanaka N and Orita K: Lateral lymph node dissection for rectal carcinoma below the peritoneal reflection. Br J Surg 81: 293-296, 1994.

21. Hida J, Yasutomi M, Fujimoto K, Maruyama T, Okuno K and Shindo K: Dose lateral lymph node dissection imprive survival in rectal carcinoma? Examination of node metastases by the clearing method. J Am Coll Surg 184: 475-480, 1997.

22. Sato H, Maeda K, Maruta M, Matsumori K and Koide Y: Who can get the beneficial effect from lateral lymph node dissection for Dukes' C rectal carcinoma below the peritoneal reflection? Dis Colon Rectum 49: 3-12, 2006. 